\title{
The effect of set on encoding and rehearsal processes in memory*
}

\author{
RICHARD A. MONTY and ROBERT KARSH \\ Behavioral Research Directorate, U.S. Army Human Engineering Laboratory, Aberdeen Proving Ground, Maryland 21005
}

and

HARVEY A. TAUB $\dagger$
Veterans Administration Hospital, Syracuse, New York 13210

\begin{abstract}
An experiment was conducted to determine the effect of manipulating S's preparation for what he will see on a given trial on his ability to mentally keep track of the number of occurrences of each of several different stimuli in a sequence. It was found that, when the same stimuli were used from trial to trial, performance was significantly better than when different stimuli were used. Foreknowledge of the specific stimuli to be used on a given trial did little to improve performance per se, but it did reduce labeling errors. The results were discussed in terms of the "spatial window" model of keeping-track performance.
\end{abstract}

It has been repeatedly demonstrated that, when Ss are required to mentally keep track of the number of occurrences of each of several different stimuli presented repetitively in a sequence, the majority of Ss mentally picture a set of windows, one corresponding to each category of stimuli presented. Individual running tallies are then kept in each window, and only the tallies are rehearsed after the presentation of each successive stimulus (Fisher \& Karsh, 1971; Monty, 1968; Monty, Taub, \& Laughery, 1965). Monty, Karsh, and Taub (1973) have shown that the "spatial window" technique implies both verbal and nonverbal (imaginal) processing. Specifically, the organizational components of the task (i.e., entering information into the appropriate bins) could be conceived as a nonverbal operation where imagery has been shown to be particularly efficient (Paivio, 1971), while rehearsal of the mental tallies in the bins would presumably be a verbal operation.

While numerous experiments obtained data that were consistent with the "spatial window" hypothesis (e.g., Fisher \& Karsh, 1971; Monty et al, 1973), none were designed to investigate the conditions that lead to the use of an imagery based strategy for encoding and rehearsal. Since studies of perceptual encoding (e.g., Haber, 1966) suggest that the more prepared $S$ is for what he will see, the more likely it is that he will have an appropriate strategy available with which to encode the stimulus, it may be hypothesized that one critical variable for the development of a spatial window strategy is the foreknowledge of the specific categories of information to be displayed on a given trial. Specifically, although previous keeping-track experiments used a wide variety of stimulus conditions,

\footnotetext{
* Requests for reprints should be sent to Richard A. Monty, Behavioral Research Directorate, Human Engineering Laboratory, Aberdeen Proving Ground, Maryland 21005.

TThis study was supported in part by the Veterans Administration as part of Project Number 8325-01. The authors gratefully acknowledge the comments of Dennis F. Fisher. This paper may be reproduced in full or in part for any purpose of the United States government.
}

all Ss within any particular group always received the same stimulus categories on all trials. Thus, complete knowledge of the stimuli to be shown and constancy of stimulus categories from trial to trial may both be necessary conditions to allow $S$ s to maintain their running tallies of stimuli without labeling and rehearsing the category names, and should lead to maximum performance. On the other hand, if stimulus categories are varied and/or knowledge of these variations withheld, keeping-track performance should be degraded, since Ss will have to encode and rehearse the stimulus names along with the tallies. Thus, the primary purpose of the present experiment was to manipulate S's preparation for what he will see on a given trial. This was done in two ways: by giving $S$ the same or different categories of information to be remembered on each trial and by giving or not giving $S$ prior information as to what these categories will be.

\section{METHOD}

Subjects

One hundred and twenty students from Harford Community College, Churchville, Maryland served as paid volunteers.

\section{Apparatus}

The apparatus has been described more fully elsewhere (Monty, Taub, \& Laughery, 1965). Briefly, S was seated in front of a panel, the face of which was positioned approximately $45 \mathrm{~cm}$ from his eyes at a 30-deg angle below his horizontal line of sight. An Industrial Electronic Engineers, Inc. in-line readout display unit was mounted in the panel. The display unit contained 12 lenses, each etched with material to be presented to the Ss. By turning on and off lights behind each of the lenses, the materials were projected onto a $3.8 \times 4.8 \mathrm{~cm}$ frosted glass surface. All sequences of stimuli and stimulus durations were preprogrammed and controlled by a Grason-Stadler 3001 SCAT system located in an adjoining room.

\section{Procedure}

The S's task was to observe and tally mentally the number of occurrences of each of four different stimuli presented sequentially and to record his tallies in writing at the end of each 


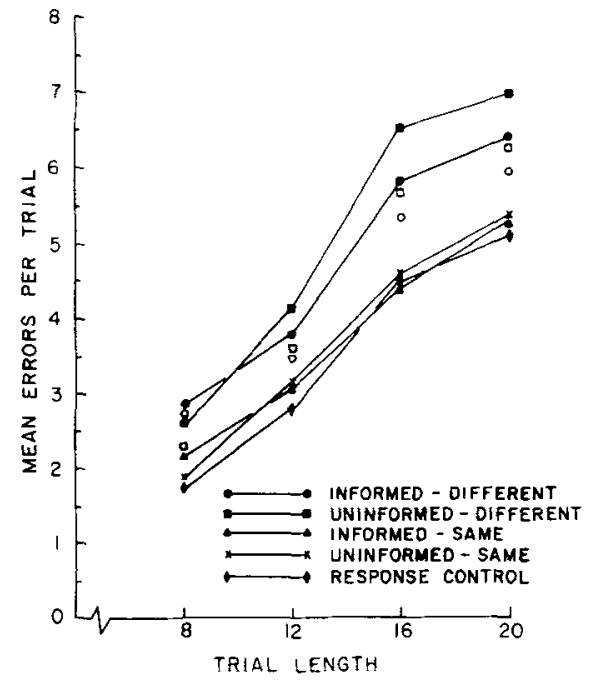

Fig. 1. Mean errors per trial as a function of trial length and groups. The open squares and circles represent the rescored data.

sequence. The population of stimuli used were the letters $P$ through $\mathrm{Z}$ consecutively. Within this population of 11 letters, there are eight possible combinations or sets of four adjacent letters (e.g., P, Q, R, S; Q R, S, T; etc.). Only one of these four-letter sets was used on any given trial. A trial consisted of a random sequence of $8,12,16$, or 20 occurrences of each of the four letters within a set, with the restriction that each letter had to occur at least once in the sequence. The letters were exposed at a rate of one every $\mathrm{sec}$, with a $0.5-\mathrm{sec}$ on time and a $0.5-\mathrm{sec}$ interstimulus off time. A $2500-\mathrm{Hz}$ tone signaled the beginning of a trial, and a similar tone signaled the end of a trial and cued $S$ s to record their tallies. The start of each trial was immediately preceded by a "pretrial" condition consisting of four letters, each exposed for $1 \mathrm{sec}$, with a 0.1 -sec off time between them. A $4000-\mathrm{Hz}$ tone signaled the beginning and end of this pretrial condition. The pretrial condition was followed by a 1-sec delay before the start of the trial per se.

The Ss were randomly assigned to five groups of 24 Ss each. The first three groups were designed to determine if prior knowledge about what was to occur on a given trial affected the Ss' keeping-track performance.

The first group (informed-different) saw a different four-letter set on each trial. Ss were informed of the specific letter to appear in each trial by being shown those letters alphabetically in the pretrial condition.

The second group (uninformed-different) saw sequences identical to those shown to the first group but were given no information as to what letters would appear in a trial. Rather, they were presented with a display of four Os during the pretrial condition.

The third group (informed-same) always saw sequences of the same four-letter set on each trial (and each pretrial condition). In order to use the entire population of letters, each of the eight four-letter sets was shown randomly to each of three Ss.

The fourth group (uninformed-same) saw the identical sequences as the third group but were shown four Os in the pretrial condition. Comparison of this group with Group 3 should reveal any differences that might result from the pretrial condition per se (i.e., relevant letters vs irrelevant Os).

Finaily, the fifth group (response control) was identical to the third group except in the manner in which Ss had to report their tallies on the answer sheet. In Groups 1, 2, 3, and 4, Ss used an unlabeled answer sheet and were required to record the letters to be tallied in addition to their tallies, but they could record them in any order they wished. In Group 5 , however, the answer sheet was prelabeled at the head of columns which required $S$ s to record the tally only, but in ordered columns. The response procedure in this group conforms to the procedures generally followed in previous studies.

Trial length, defined as the total number of stimuli to be kept track of in a sequence, was included as a within-Ss variable. All Ss were presented with trial lengths of $8,12,16$, and 20 stimuli. Each of the trial lengths was presented twice in random order during each of the four blocks of eight trials. Similarly, each of the eight possible sets of letters was presented once per block in a random fashion, with the restriction that within each block a specific letter set occurred once and only once at any trial length.

\section{RESULTS}

The sum of the absolute difference between the number of letters shown in each category and the number reported in each category was used as the principal measure of performance (Monty, Taub, \& Laughery, 1965). For example, if 5 Qs, 3 Rs, 2 Ss, and 2 Ts were shown on a given trial and $\mathrm{S}$ reported $7 \mathrm{Qs}, 2$ Rs, 2 Ss, and $2 \mathrm{Ts}$, then the error for that trial would be 2 for the Qs and 1 for the Rs, for a total of 3. Further, given the same presentation, should $S s$ have reported the wrong category names as, for example, $7 \mathrm{Qs}, 1 \mathrm{R}, 3 \mathrm{Vs}$, and $1 \mathrm{~W}$, the error for that trial would be 2 for Qs, 1 for Rs, 2 for Ss, and 2 for $T s$, for a total of 7.

The absolute error scores for the two equal trial lengths within each block were summed and subjected to ąn analysis of variance (Butler, Kamlet, \& Monty, 1969) with groups as a between effect and trial lengths and blocks as within effects. Each of the main effects reached significance, indicating that performance fluctuated slightly, but in no meaningful pattern, across blocks $[F(3,345)=3.42, p<.05]$, deteriorated with an increase in trial length $[\mathrm{F}(3,345)=403.42, \mathrm{p}<.001]$, and differed as a function of groups $[F(4,115)=7.60$, $p<.01]$. The Blocks by Trial Length interaction also reached significance $[F(9,1035)=5.66, p<.01]$, simply indicating minor fluctuations in the trial length effect from block to block.

Of greater interest is the significant Groups by Trial Length interaction $[\mathrm{F}(12,345)=2.33, \mathrm{p}<.01]$. The data underlying this interaction are shown in Fig. 1. This interaction was evaluated further with Scheffé tests run at each trial length. The results of these tests indicate that there were no differences between groups at a trial length of 8 and that the magnitude of the differences rose to a maximum at a trial length of 16 , with the uninformed-different and informed-different groups each being significantly poorer in performance $(p<.05)$ than each of the three groups that always received the same categories. However, none of the comparisons suggested any significant differences between the uninformed-different and the informed-different groups. Thus, these analyses suggest that, when Ss view different letters on each trial, performance is poorer than if they view the same letters. Further, providing Ss with foreknowledge of the letters they are about to see on a given trial does not appear to bring about significant 
improvements in performance. Finally, these results do not appear to be due merely to the requirement for writing or recording the category names, since the group that received the same letters on each trial but had to record only their tallies (response control group) was not affected significantly and performed in a fashion similar to the other groups that received these same categories.

To determine if some of the above conclusions were due to the choice of scoring criteria that did not discriminate between labeling errors and keeping-track errors, the data for the two groups receiving different stimuli on each trial was rescored in the same fashion, except that the erroneous labels frequently recorded by the two groups receiving different stimuli on each trial were treated as correct. For example, if $\mathrm{S}$ saw $5 \mathrm{Qs}, 3$ Rs, 2 Ss, and 2 Ts but reported 7 Qs, 2 Rs, 2 Ss, and 2 $\mathrm{Ws}$, the $\mathrm{W}$ would have been scored as a $\mathrm{T}$; thus, there would be no errors for Ts. On those occasions when $\mathrm{S}$ mislabeled two categories on the same trial, he was given the best score that could result by assignment of the correct labels. In no instance were there more than two categories incorrectly reported on the same trial. These scores were resubjected to an analysis of variance identical to the first analysis presented previously. As in the previous analysis, the main effects for blocks $[F(3,345)=3.37, \quad p<.05]$ and trial length $[F(3,345)=385.90, p<.001]$, and the Block by Trial Length interaction $[F(9,1035)=5.40, p<.001]$ all reached significance and revealed the same trends noted above. Of greater importance is the significant main effect for groups $[F(4,115)=3.49, p<.05]$ coupled with the failure of all interactions with groups to reach significance. The data for the informed-different and uninformed-different groups underlying this interaction are shown by the open circles and squares in Fig. 1. It can be seen, as would be expected, that the rescoring resulted in a substantial drop in the number of errors. The important point to be made, however, is that the Scheffé test indicated that the two groups receiving the different stimuli on each trial did not differ in their performance. Further, the combined performance of these two groups was significantly poorer than that of the three groups receiving the same stimuli on each trial $(p<.05)$. Thus, the same-different breakdown leads to more than the simply erroneous labeling. It apparently also leads to a breakdown in keeping-track performance per se.

Finally, since the lack of difference between the uninformed-different and the informed-different groups could also be explained by a failure to use the information provided at the beginning of each trial, the number of categories labeled improperly by these groups were examined. The data were similarly subjected to an analysis of variance, with trial length and blocks as within effects and groups as a between effect. In addition to a significant effect for groups $[F(1,46)=5.40, p<.05]$, blocks $[F(3,138)=8.07$, $\mathrm{p}<.001]$, and the Blocks by Trial Length interaction
$[F(9,414)=2.30, p<.05]$, the Groups by Blocks by Trial Length interaction reached significance $[F(9,414)=2.35, p<.05]$. The data underlying the latter interaction indicated that, while the informed group performed considerably better than the uninformed group over all conditions (mean number of categories mislabeled was 0.42 and 0.60 , respectively), the magnitude of the difference varied widely as a function of both trial lengths and blocks. Thus, it appears that, while foreknowledge had no consistent effect upon keeping-track performance per se, it seemed to reduce the likelihood of labeling errors.

\section{DISCUSSION}

Taken together, the results indicate that the lack of constancy of stimulus categories from trial to trial results in two kinds of errors: those stemming from the mislabeling of categories and keeping-track errors per se. Labeling errors reflect the tendency of Ss to mislabel the categories on their answer sheet by reporting category names shown on earlier trials. However, even when such errors are taken into account, there remains a substantial error in tallying, i.e., keeping-track performance per se. Foreknowledge of the specific category to be shown on a given trial effectively reduces the labeling errors but not the keeping-track errors per se. In other words, foreknowledge seems to improve Ss' ability to remember which categories were presented on a given trial but not their ability to tally. Use of the same categories on each trial completely eliminates labeling errors, as would be expected, but also improves Ss' ability to tally mentally. Further, the magnitude of labeling errors relative to keeping-track errors is quite small.

In Paivio's terms (1971), the spatial window technique implies both verbal and nonverbal (imaginal) processing. The organizational components of the task (i.e., entering information into the appropriate bins) would be conceived of as a nonverbal operation where imagery has been shown to be particularly efficient, while rehearsal of the mental tallies in the bins would presumably be a verbal operation. In terms of the present experiment, the finding that the magnitude of labeling errors is quite small relative to the keeping-track errors per se might imply that, in the absence of constancy of categories from trial to trial, Ss rehearse category names in addition to the tallies in their windows or bins. This technique would tend to slow the rehearsal process, thereby resulting in the noted increase in keeping-track errors per se. Such a technique would also account for the relatively small number of labeling errors. In short, then, it is hypothesized that the lack of constancy of categories from trial to trial interferes with the nonverbal processing (i.e., imagery) and forces Ss to revert to less efficient verbal operations.

Finally, it should be mentioned in passing that the observation that the response control condition, in which the Ss were required to record only the tallies and 
not the category names, did not differ from the two groups in which they had to record both the tallies and the names is an important one. It suggests that manipulating the manner in which the material to be remembered must be reported does not interact with the efficiency with which Ss perform the keeping-track task itself.

\section{REFERENCES}

Butler, D. H., Kamlet, A. S., \& Monty, R. A. A multipurpose analysis of variance FORTRAN IV computer program. Psychonomic Monograph Supplements, 1969, 2(Whole No. 32), 301-319.

Fisher, D. F., \& Karsh, R. Modality effects and storage in sequential short-term memory. Journal of Experimental Psychology, 1971, 87, 410-414.
Haber, R. N. Nature of the effect of set on perception. Psychological Review, 1966, 73, 335-351.

Monty, R. A. Spatial encoding strategies in sequential short-term memory. Journal of Experimental Psychology, 1968, 77, 506-508.

Monty, R. A., Karsh, R., \& Taub, H. A. Imagery and interference in sequential short-term memory. Canadian Journal of Psychology, 1973, 27, 220-226.

Monty, R. A., Taub, H, A., \& Laughery, K. R. Keeping track of sequential events: Effects of rate, categories, and trial length. Journal of Experimental Psy chology, 1965, 69, 224-229.

Paivio, A. Symbolic and sensory modalities of memory. Research Bulletin No. 216, Department of Psychology, University of Western Ontario, London, Canada, 1971.

(R eceived for publication March 4, 1974; accepted April 1, 1974.) 